\title{
AS INOVAÇÕES TECNOLÓGICAS LEVAM O JORNALISMO AOS AGENTES AUTÔNOMOS ARTIFICIAIS
}

\section{Technological innovations carry journalism to artificial and autonomous agents}

\author{
Innovaciones tecnologicas llevam el periodismo a los agentes independientes \\ artificiales
}

\begin{abstract}
Sebastião Carlos de Morais Squirra Doutor pelo Programa de Pós-Graduação em Ciências da Comunicação da Escola de Comunicação e Artes da Universidade de São Paulo. ssquirra@gmail.com
\end{abstract}

\author{
Krishma Anaísa Coura Carreira \\ Jornalista e doutoranda no Programa de Pós-Graduação em Comunicação Social \\ da Universidade Metodista de São Paulo. \\ krishmacarreira@gmail.com
}

\section{Resumo}

Nos últimos anos, inovações tecnológicas disruptivas inseriram radical tecnologização nos recursos comunicativos de massa, fazendo com que parte significativa da sociedade aderisse de forma consistente à sua utilização. Tal transformação alterou fortemente os processos produtivos do Campo da Comunicação, especialmente no segmento do Jornalismo, onde se consolidou uma densa automatização dos processos e, mais recentemente, Agentes autônomos artificiais passaram a apurar, redigir e distribuir notícias sem a participação humana. Neste trabalho abordamos a incorporação de sistemas de Inteligência Artificial à realidade do Jornalismo, o que está reconfigurando as lógicas produtivas e requerendo novas competências profissionais. As reflexões deste artigo se baseiam em uma ampla revisão bibliográfica de caráter exploratório e interdisciplinar

Palavras-chave: Jornalismo automatizado. Tecnologias disruptivas no jornalismo. Jornalismo e inteligência artificial.

\begin{abstract}
In recent years, disruptive technological innovations have introduced radical technologizing in mass communicative resources, making a significant part of society consistently adhere to its use. This transformation has greatly altered the productive processes of field of Communication, especially in the Journalism segment, where a dense automation of processes has been consolidated and, more recently, Artificial autonomous agents began to compose, to write and distribute news without human participation. In this work we approach the incorporation of Artificial Intelligence systems to the reality of Journalism, which is
\end{abstract}


reconfiguring the productive logics and requiring new skills and professional skills. The reflections of this article are based on an extensive bibliographical review of an exploratory and interdisciplinary character.

Key words: Automated journalism. Disruptive technologies in journalism. Journalism and artificial intelligence.

\section{Resumen}

En los últimos años, las innovaciones tecnológicas disruptivas han introducido una radical tecnología en los recursos comunicativos masivos, haciendo que una parte significativa de la sociedad se adhiera de forma consistente a su utilización. Esta transformación alteró fuertemente los procesos productivos del Campo de la Comunicación, especialmente en el segmento del Periodismo, donde se consolidó una densa automatización de los procesos y, más recientemente, Agentes autónomos artificiales pasaron a averiguar, redactar y distribuir noticias sin la participación humana. En este trabajo abordamos la incorporación de sistemas de Inteligencia Artificial a la realidad del Periodismo, lo que está reconfigurando las lógicas productivas y requiriendo nuevas competencias profesionales. Las reflexiones de este artículo se basan en una amplia revisión bibliográfica de carácter exploratorio e interdisciplinario.

Palabras clave: Periodismo automatizado. Las tecnologías de punta en el periodismo; El periodismo y la inteligencia artificial.

\section{INTRODUÇÃO}

A informação jornalística é um recurso comunicativo massivamente adotado nas sociedades. Nesse contexto, as múltiplas formas do fazer jornalístico sempre se caracterizaram por serem estruturadas em largos e consistentes sistemas e processos tecnológicos. No presente trabalho, revisitamos conceitos que sugerem o alargamento e a consolidação de uma plena tecnologização no fazer jornalístico. Isso, pois tal alteração processual abarcou uma inimaginável automatização nos processos da produção e disseminação da informação, desde o levantamento, organização e seleção de dados, sua estocagem e distribuição, mas sobretudo a própria elaboração dos relatos, uma vez que recentemente foram inseridos recursos automatizados para a redação de notícias.

Alterações nos processos produtivos com a contínua tecnologização do jornalismo vêm acontecendo desde o final do século passado, principalmente após a criação da rede mundial de computadores e das plataformas e aplicativos digitais que foram adotados. Os avanços na área da Inteligência Artificial e o emprego crescente de algoritmos sofisticados pelas organizações jornalísticas de países da América do Norte, Europa e Ásia indicam que tal modelagem pode ser adotada nas demais nações. 
Neste artigo não seguimos uma visão neutra da tecnologia. Muito menos uma concepção determinista, segundo a qual a "tecnologia causa ou determina a estrutura do resto da sociedade e da cultura". Também não temos uma visão da tecnologia como autônoma, no sentido de que ela "não está sob o controle humano" e que tem uma lógica própria (DUSEK, 2009, p.117). Trabalhamos a partir de uma visão sistêmica, que inclui o contexto, a intencionalidade, o instrumental, "as habilidades e organização humanas necessárias" para operar a tecnologia e mantê-la (p.50). Portanto, o desenvolvimento de softwares de automação de parte das atividades jornalísticas não é garantia de que o produto obtido a partir deles será apropriado ou terá resultados positivos para a humanidade.

\section{A CRESCENTE TECNOLOGIZACÃO DO JORNALISMO}

Entendemos que a relação da comunicação com a tecnologia é antiga, sendo a própria escrita um dos primeiros exemplos. A partir do momento que a palavra passou a ser representada na pedra e depois no papel, configurou-se como produto de ferramentas, tornando-se "ela própria uma ferramenta" (GLEICK, 2013, p.39), portanto, tecnologia. A prensa de tipos móveis de Johannes Gutenberg é outra tecnologia cujo uso impactou na disseminação do conhecimento, que era restrita a grupos pequenos. Relatos em folhas e volantes começaram, então, a circular e, no final do século 17, foram substituídos por publicações regulares e o jornalismo tornou-se uma prática social consistente (RÜDIGER, 2003, p.15).

Até 1836, as comunicações escritas eram transportadas de um lugar para outro e enfrentavam muitos problemas (distância, dependiam de cavalos, barcos e até da boa vontade e do compromisso do entregador), o que sempre foi um desafio para a comunicação (COSTELLA, 2001). No final do século 19, todos os continentes foram ligados pelo telégrafo e, neste cenário, a informação passou a ser uma mercadoria consumida mundialmente, sendo coletada, produzida e distribuída em escala industrial, quando as agências de notícias surgiram para administrar esse novo fluxo informativo.

Em 1876, surge o telefone, instrumento que é "ao mesmo tempo, um dos mais antigos meios de comunicação e o mais atualizado. Muitas das tecnologias que hoje revolucionam o mundo têm sua origem no sistema de telefonia" (STRAUBHAAR; LAROSE, 2004, p.152). Com o telégrafo e o telefone ocorreu o salto da infraestrutura de uma comunicação 
unicamente física para "uma outra baseada em transmissão eletrônica - primeiro por fios, daí muitos anos depois, também por ondas de rádio (p.41).

Novo salto tecnológico ocorreu em 1948 com a invenção do transístor, um pequeno semicondutor eletrônico criado nos Laboratórios Telefônicos da Bell (a mesma empresa responsável pela existência do telefone), nos Estados Unidos. Para Gleick (2013, p.12), “o transístor deu início a uma revolução na eletrônica, colocando a tecnologia no rumo da miniaturização e da onipresença". No mesmo Laboratório, Claude Shannon criou um neologismo para denominar a unidade básica de informação que, portanto, passaria a ser mensurada. Foi assim que nasceu o bit e, com ele, as bases para a futura revolução digital, quando estratégias militares do final da Segunda Guerra Mundial levaram ao desenvolvimento do Colossus, um computador construído para decifrar códigos nazistas e do Eniac, projetado para calcular a trajetória de balas de canhões e mísseis.

Durante a Guerra Fria, nas décadas de 1950 e 1960, outras ações lançaram as bases da internet quando pesquisadores norte-americanos desenvolveram uma rede de conexões, descentralizada e sem nenhum tipo de hierarquia, para comunicação em caso de ataque atômico. A arquitetura deste sistema de comunicação levou à criação da rede mundial de computadores, quando na transição para 1990, o físico inglês Tim Berners-Lee ajudou a desenvolver a World Wide Web (WWW) no Centro Europeu de Pesquisas Nucleares (Cern). Com uma arquitetura aberta e de fácil funcionamento para o público sem conhecimento técnico, a web passou a permitir a troca de informações entre quaisquer computadores conectados à rede.

Essa transformação tecnológica atingiu plenamente o ecossistema da comunicação, especialmente o jornalístico, pois meios como rádio, televisão e jornal impresso passaram a migrar e a se integrar com os computadores. Até então cada meio tinha suportes específicos e as tecnologias digitais passaram a permitir que as potencialidades das mídias fossem mescladas. Tudo ficou convergente: o conteúdo, os mercados midiáticos e até os públicos dos meios de comunicação, que passaram a ir "a qualquer parte em busca das experiências de entretenimento que desejam" (JENKINS, 2009, p.29), alterando também as formas narrativas.

Segundo Lev Manovich (2001), a linguagem das novas mídias se estrutura em 5 princípios: a) representação numérica através de bits (o que faz com ela seja programável ou manipulável); b) modularidade (pode ser reorganizada em um número infinito de combinações); c) automação (tarefas podem ser executadas automaticamente pela máquina, tomadas de acordo com informações fornecidas pelo usuário; d) variabilidade (fluxo 
ininterrupto, possibilidade de mudança constante) e e) transcodificação (transformação de mensagens de qualquer meio em dados de computador).

Luís Mauro Sá Martino (2015, p.11) descreve ainda outros conceitos-chave das mídias digitais: a) criação de um ciberespaço (espaço de interação criado no fluxo de dados digitais em redes de computadores, "virtual por não ser localizável no espaço, mas real em suas ações e efeitos"); cultura participativa (qualquer pessoa pode ser um produtor de conteúdo); c) interatividade (interação entre usuários ou entre "usuários, programas e conteúdos"); d) interface (a operação das mídias digitais a "partir de pontos de contatos 'amigáveis' entre dispositivos e usuários"); e) segurança e vigilância (possibilidade de identificar dados e redefinição de privacidade); f) ubiquidade; g) velocidade e h) virtualidade (mídias sociais são independentes de ambiente físico).

As tecnologias aqui citadas (prensa, telégrafo, telefone, computadores, internet e web) vêm sendo exaustivamente usadas pelo jornalismo, sendo um dos elementos que ajudaram a romper alguns processos rotineiros pelos quais as notícias eram produzidas (SAYURI, 2014). Mas vale ressaltar que algumas práticas e características da era analógica ainda convivem na era digital (PRIMO, 2011, p. 141).

\section{BIG DATA, INTELIGÊNCIA ARTIFICIAL E JORNALISMO}

Com as tecnologias digitais, o processo de apuração da notícia mudou radicalmente, uma vez que os dados passaram a ser obtidos através das redes telemáticas e da estruturação de bancos de dados digitais para a criação e enriquecimento de relatos confiáveis (SQUIRRA, 2013, p.73). O Jornalismo Digital em Base de Dados (JDBD) tornou-se um paradigma, que é descrito por Suzana Barbosa como o modelo que tem as bases de dados como definidoras da estrutura e da organização, além da apresentação dos conteúdos de natureza jornalística, de acordo com as funcionalidades e categorias específicas, que vão permitir a criação, a manutenção, a atualização, a disponibilização e a circulação de produtos jornalísticos digitais dinâmicos (2007, p. 28).

Os dados digitais são gerados por variados tipos de registro, atividades e transações através de softwares e redes de computadores, em dispositivos fixos ou móveis, de uso em redes sociais, dos arquivos on-line públicos, governamentais, empresarias, educacionais, dos sensores químicos e atmosféricos, de câmeras de segurança e através de formatos diversos como áudio, audiovisual ou texto, etc. Nos anos 90 do século 20 começou a ser usado o 
conceito de Big Data como um novo jeito de armazenar, gerenciar e analisar grandes volumes de dados estruturados de diversas fontes, em uma velocidade considerável. Big Data demanda a utilização de técnicas, processos e ferramentas mais complexos para encontrar valor e transformar dados em informações confiáveis e relevantes, que vão ajudar na tomada de decisões e na geração de insights.

A Internet das Coisas ou dos Objetos, também conhecida pela sigla IoT do inglês Internet of Things, tem ajudado ainda mais no crescimento do volume de dados digitais. Ela foi impulsionada a partir de 1999 com o trabalho de um grupo conhecido por Auto-ID Center, que se dedicava à identificação de frequência de rádio em rede (RFID) e às tecnologias de sensores inteligentes dentro do Instituto de Tecnologia de Massachusetts (MIT Massachussets Institute of Technology). A partir dele, foi criado o Auto-ID Labs - uma junção de seis universidades: o próprio MIT, a Universidade de Cambridge (University of Cambridge), a Universidade de Adelaide (University of Adelaide), a de Keio (Keio University), de Fudan (Fudan University) e de St. Gallen (University of St. Gallen).Cada uma destas universidades tinha seus próprios interesses, mas em comum, todas miravam na meta de desenvolvimento de uma arquitetura padrão para criar uma rede global contínua de objetos físicos usando a RFID.

Foi assim que começou a se projetar uma Internet das Coisas, que nada mais é do que a conexão dos objetos (carros, geladeiras, celulares, etc.) à internet. De acordo com o Cisco Internet Business Solutions Group (IBSG), a Internet das Coisas só pode ser considerada uma realidade a partir do momento em que estão conectados à internet mais objetos do que pessoas (EVANS, 2011, p.2). Em 2003, havia cerca de 6,3 bilhões de pessoas no mundo e 500 milhões de dispositivos conectados. Em 2010, enquanto existiam 6,8 bilhões de seres vivos, 12, 5 bilhões de dispositivos estavam conectados à rede. Portanto, a Internet das Coisas deve ter nascido de fato entre 2008 e 2009. Segundo a consultoria ICD, até 2020 devem existir 29,5 bilhões de objetos ligados à rede (KATO, 2015, p.4).

Dentro do universo digital, os algoritmos são uma das ferramentas usadas no processo de converter dados em informação. Eles são sequências de instruções adotadas para resolver problemas ou tarefas. Essa estrutura de cálculos ajuda a máquina a "tomar decisões, exibir resultados e, se necessário, lidar com esses resultados novamente, dando início a outra rodada de operações matemáticas" (MARTINO, 2015, p.213). Os algoritmos são usados no jornalismo para minerar grandes volumes de dados com o objetivo de prever determinados fatos, como resultado de eleições, por exemplo, cujo resultado seria impossível de ser 
alcançado sem as ferramentas do Big Data, ou demandaria um tempo enorme de um grande time de jornalistas.

Outro algoritmo, capaz de identificar padrões ocultos e de construir previsões foi usado, por exemplo, em 2014, pelo jornalista Nate Silver, do blog Five Thirty Eight do The New York Times, quando este jornalista conseguiu adiantar com precisão o resultado das eleições para o senado norte-americano. Outros podem ser empregados na análise de determinados ambientes, como redes sociais, como aconteceu em 2011, quando a equipe do britânico The Guardian usou algoritmos descritivos para identificar, no Twitter, como os tumultos nas manifestações de Tottenham se espalhariam. Alguns algoritmos também podem cumprir a tarefa de identificar anomalias ou valores "fora da curva" e programas com este objetivo são usados no jornalismo investigativo, que atua como uma espécie de fiscal de outros poderes, função também conhecida no meio jornalístico como "cão de guarda". Certos algoritmos podem ser projetados para analisar a Deep Web, uma gigantesca área da internet, invisível aos mecanismos de busca tradicionais e que é bem maior que a web visível.

Estes são alguns exemplos da profunda alteração na arte da apuração e produção de notícias que, para usar a expressão de Michael Dertouzos (2002, p. 89), vive uma revolução 'inacabada'. Essas mudanças são pequenos indicadores "do que está por vir" (BRYNJOLFSSON; MCAFEE, 2015, p.41). Os limites do mundo digital são muito flexíveis permitindo que os dispositivos informatizados exponenciem as capacidades que possuem, tornando-se mais rápidos, menores, baratos e mais eficazes. A tecnologia digital "permite fazer coisas que, anteriormente, pareciam fora de alcance" (BRYNJOLFSSON; MCAFEE, 2015, p.57). Uma dessas possibilidades, impensada até há pouco tempo, é a automatização de algumas funções do processo jornalístico a partir de avanços da Inteligência Artificial.

O termo inteligência artificial (IA) foi usado pela primeira vez em 1956, em uma Conferência no Dartmouth College, nos EUA. De acordo com a definição contida na Associated for Computing Machinery (ACM), IA é um campo da ciência da computação e da engenharia interessado em comportamento inteligente e na criação de artefatos que exibam este comportamento. Para Ray Kurzweil (2007 p. 412), a IA trabalha com sistemas baseados em conhecimentos como "sistemas especialistas, reconhecimento de padrões, aprendizado autônomo, compreensão de linguagem natural, robótica e outras".

Destacamos que na definição de inteligência humana há um objetivo que também é comum ao conceito de algoritmo, pois ela pode ser entendida como "a capacidade, mais ou menos grande, de resolver um problema" (COMTE-SPOVILLE, 2003, p. 318) e o algoritmo 
representa exatamente uma sequência de instruções para solucionar uma tarefa. Mas o pesquisador Blay Whitby (2004, p.120) reforça que não é apropriado aplicar um viés antropocêntrico à ideia inteligência artificial. "A IA é genuinamente artificial. As pequenas e grosseiras tentativas de conseguir reproduzi-la que foram feitas até agora sugerem que ela é muito diferente da inteligência natural. Entretanto, ainda assim é inteligência". Depois de mais de 50 anos e com períodos de altos e baixos em face dos problemas provocados pelas expectativas exageradas e com resultados limitados (KAKU, 2011), o campo da IA vem avançando consistentemente nas últimas décadas, como na área do Jornalismo, que é o objeto de análise deste artigo.

Neste trabalho, incorporamos o termo "Agentes autônomos artificiais", conforme sugerido por Gudwin (2016), como sinônimo de softwares de geração de linguagem natural ou plataforma de geração de linguagem natural (GLN). A GLN é um sistema de computador que, a partir de dados, produz, automaticamente, um texto em linguagem humana (KAA; KRAHMER, 2014, p.1). Com a explosão de dados disponíveis, com o aumento da importância das análises estatísticas, da inclusão de conhecimentos linguísticos e de novas técnicas mais precisas de geração de textos, a GLN avançou muito e algumas pesquisas sinalizam que, no caso do jornalismo, nem sempre os leitores - inclusive alguns jornalistas percebem que uma matéria foi redigida por um computador (CLERWALL, 2014; KAA, KRAHMER, 2014; GRAEFE et al., 2016).

\section{NOTÍCIAS AUTOMATIZADAS: POTENCIAIS, LIMITES E CONSEQUÊNCIAS}

A automação representa a forma mais radical de criação de conteúdos, pois nela a ação principal se dá a partir dos algoritmos, sendo que a participação humana fica restrita ao desenvolvimento do código de programação. Na opinião de Haak, Parks e Castells (2012) o jornalismo automatizado (automated journalism) integra uma lista de tendências dessa atividade profissional: o jornalismo em rede (networked journalism); a inteligência coletiva e conteúdo gerado por usuários (crowdsourcing and user-generated content); a mineração, análise, visualização e mapeamento de dados (data mining, data analysis, data visualization and mapping); o jornalismo de 'ponto de vista' (point of view journalism) e o jornalismo global (global journalism).

No jornalismo, os agentes autônomos artificiais ou softwares de geração de linguagem natural passaram a executar, de forma automática, um conjunto de funções antes feitas, nas 
redações, por jornalistas e que já foram sistematizadas pela teoria do newsmaking como recolha de dados, seleção e apresentação das notícias (WOLF, 1987). Eles ainda podem distribuir o conteúdo em diversas plataformas e redes sociais (LOKOT, DIAKOPOULOS, 2015) e adaptar o conteúdo para qualquer formato de tela e dispositivo. O professor Márcio Carneiro dos Santos (2016) alerta para a necessidade de não confundir o produto jornalístico apurado e redigido automaticamente pelas plataformas de GLN com o Jornalismo Digital em Base de Dados - JDBD, uma vez que "os algoritmos de inteligência artificial (IA), que suportam as narrativas automatizadas [...] pertencem a uma categoria diferente e não devem ser confundidos com outras, principalmente pela lógica de procedimentos específica sob a qual operam". Se existe um consenso sobre a definição do que é a automação nos sistemas de apuração, produção e circulação de notícias, ainda não há um acordo quanto à terminologia adotada, como pode ser observado no quadro 1.

Quadro 1 - Termos usados para definir as notícias produzidas automaticamente

\begin{tabular}{|l|l|l|}
\hline \multicolumn{1}{|c|}{ Autor (ano) } & \multicolumn{1}{|c|}{$\begin{array}{c}\text { País/vínculo } \\
\text { com a } \\
\text { universidade }\end{array}$} & \multicolumn{1}{|c|}{ Termo } \\
\hline DALEN (2012) & Dinamarca & Robot journalism \\
\hline CLERWALL (2014) & Suécia & Robot journalism \\
\hline LATAR (2014, 2015) & Israel & Robot journalism \\
\hline ALJAZAIRI (2016) & Suécia & Robot journalism \\
\hline KIM, D.; KIM, S. (2016) & Coreia do Sul & Robot journalism \\
\hline HAAK; PARKS; CASTELLS (2012) & Holanda/EUA & Automated journalism \\
\hline KAA; KRAHMER (2014) & Holanda & Automated journalism \\
\hline LECOMPTE (2015) & EUA & Automated journalism \\
\hline CARLSON (2015) & EUA & Automated journalism \\
\hline GRAEFE (2016) & Alemanha & Automated journalism \\
\hline
\end{tabular}


PROGRAMA DE PÓS-GRADUAÇÃO EM COMUNICAÇÃO DA UNIVERSIDADE FEDERAL DE SANTA MARIA

\begin{tabular}{|l|l|l|}
\hline GRAEFE et al. (2016) & Alemanha & Automated journalism \\
\hline SANTOS (2016) & Brasil & Jornalismo automatizado \\
\hline DIAKOPOULOS (2013) & EUA & Algorithmic journalism \\
\hline DÖOR (2015) & Suíça & Algorithmic journalism \\
\hline ANDERSON (2013) & EUA & Algorithmic journalism \\
\hline ANDERSON (2013) & EUA & $\begin{array}{l}\text { Algorithmic news / automated } \\
\text { content }\end{array}$ \\
\hline NORMANDE (2013) & Brasil & Narrativas automatizadas \\
\hline LINDEN (2016) & Finlândia & Computational journalism \\
\hline COSTA (2015) & Brasil & Reportagem algorítmica \\
\hline
\end{tabular}

Fonte: CARREIRA (2017)

Não foram adotados os termos algorithmic journalism, algorithmic news e computational journalism porque eles não fazem uma conexão direta com a ideia de automação da notícia.

Automated content e narrativas automatizadas também foram descartados porque não exibem uma referência direta ao Jornalismo. Não usamos o termo jornalismo robótico porque o jornalismo não pode ser feito realmente por robôs. Apesar da expressão jornalismo automatizado (automated journalism) ser adequada de acordo com nossa análise, uma vez que todas as etapas do processo de produção podem ser automatizadas, preferimos usar o termo Notícias Automatizadas (ainda não empregado por outros pesquisadores) porque do ponto de vista de produto ou gênero jornalístico, somente a notícia pode ser automatizada até o momento. Em função desta concepção, também decidimos não usar reportagens algorítmicas, pois as reportagens ainda não são automatizadas. (CARREIRA, 2017, p.105).

As reportagens não podem ser produzidas pelos Agentes autônomos artificiais porque elas demandam uma operação mais "analítica ou crítica da realidade, o que exige alto grau de subjetividade, algo, portanto, pouco propício à automação" (ARCE, 2009, p.4). Os softwares também não são capazes de explicar fenômenos (GRAEFE, 2016), característica essencial das reportagens. As notícias automatizadas só podem ser usadas na cobertura de assuntos baseados em dados estruturados, que são aqueles organizados e armazenados em bases de 
dados, em linhas e colunas de planilhas, formulários, etc. Por isso, as notícias automatizadas são mais comuns em assuntos muito baseados em dados, como esportes, finanças (conforme a figura 1), resultados eleitorais, previsão de tempo, entre outros tópicos.

\section{EPS Estimates Down for J.M. Smucker In Past Month}

$\begin{aligned} & \text { Narrative Science, } \\ & \text { Fult BIO } \checkmark \\ & \text { opinions expressed by forbes contributors are their own. }\end{aligned}$
Over the past three months, the consensus estimate has sagged from
\$1.25. For the fiscal year, analysts are expecting earnings of $\$ 5.75$ per
share. A year after being $\$ 1.37$ billion, analysts expect revenue to fall $1 \%$
year-over-year to $\$ 1.35$ billion for the quarter. For the year, revenue is
expected to come in at $\$ 5.93$ billion.
A year-over-year drop in revenue in the fourth quarter broke a three-
quarter streak of revenue increases.
The company has been profitable for the last eight quarters, and for the
last four, profit has risen year-over-year by an average of $16 \%$. The
biggest boost for the company came in the third quarter, when profit
jumped by $32 \%$.
Earnings estimates provided by Zacks.
Narrative Science, through its proprietary artificial intelligence
platform, transforms data into stories and insights.

Figura 1 - Texto produzido automaticamente pela plataforma Narrative Science no site da Forbes Fonte: forbes.com

Os Agentes autônomos artificiais são programados para transformar os dados em linguagem adequada ao estilo de cada de empresa de jornalismo. Eles avaliam os dados a partir de determinados parâmetros estabelecidos; identificam o que é notícia; estruturam e escrevem a matéria, escolhem ou evitam palavras e até usam sinônimos para evitar repetições. Entretanto, os softwares de GLN redigem textos com certas limitações no que se refere às nuances da linguagem humana, como humor, metáforas e sarcasmo.

A automação de notícias começou a se tornar possível no final da primeira década do século 21. Ela já está sendo empregada em pelo menos 62 empresas de jornalismo, como Reuters, Le Monde, Xinhua, Yandex, Washington Post, Bloomberg, Associated Press e é desenvolvida por equipes internas dos grupos de comunicação ou por empresas de tecnologia nos EUA, Alemanha, França, Israel, Reino Unido, Coreia do Sul, China, Rússia, Noruega, Dinamarca e Suécia. O número mapeado até agora pode ser bem maior, pois nem todas as empresas divulgam que estão distribuindo notícias automatizadas. Além disso, a automação já se espalhou por diversos outros locais através de agências de notícias, uma vez que os países 
citados se referem unicamente à localização das sedes das empresas e não ao raio de ação delas. Importante ressaltar que no Brasil, a automação de textos está sendo empregada, mas ainda não dentro de redações.

Nas empresas de comunicação com notícias automatizadas passa a ocorrer uma reestruturação das rotinas produtivas, com uma divisão de tarefas entre os jornalistas e os Agentes autônomos artificiais. Tudo indica que jornalista humano e o software se tornarão cada vez mais integrados e formarão um casamento homem-máquina (GRAEFE, 2016) A Associated Press (AP) começou a publicar notícias automatizadas sobre resultados financeiros acumulados pelas companhias públicas norte-americanas em julho de 2014. A AP elaborou um estudo onde sugere um novo fluxo de trabalho, na redação, com os softwares de Inteligência Artificial, conforme demonstra a figura 2.

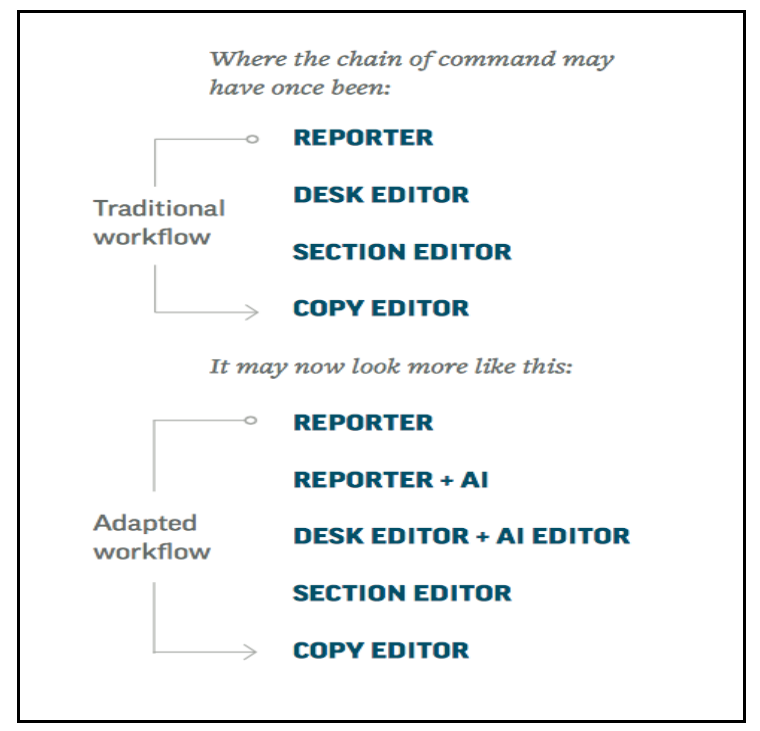

Figura 2: Novo fluxo de trabalho com automação de notícias (Associated Press)

Fonte: (MARCONI, SIEGMAN, 2017)

Diante deste novo fluxo de trabalho, torna-se necessário definir se é um jornalista ou um Agente autônomo artificial que fará determinada matéria. A escolha será determinada pelas habilidades de cada um. Vamos nos ater aos momentos em que a automação pode ser uma opção. Uma das vantagens dos softwares é que eles podem fazer várias versões simultâneas (estilo de texto e língua) a partir dos mesmos dados. A empresa Automated Insights tem uma plataforma que produz automaticamente notícias para a AP e para o Yahoo. No site da empresa aparece a informação de que a partir de um único texto com 3 sentenças, o software pode gerar 140.000 possibilidades de variações. O exemplo apresentado é um texto de e-commerce, mas o mesmo pode ocorrer com uma matéria jornalística. Essa possibilidade 
pode ajudar na ampliação da base de leitores de um veículo em função de uma maior personalização da notícia, o que pode ter, inclusive, impactos sobre monetização e modelo de negócios. O software de automação também consegue, a partir do imenso volume de dados digitais estruturados, identificar tendências, padrões e fazer previsões a partir deles. Assim, segundo Linden (2016, p.11), os processos editoriais jornalísticos podem passar a ter ações mais preditivas do que meramente reativas.

Além disso, softwares também são válidos para produzir notícias de tópicos muito descritivos e relatos curtos (GRAEFE, 2016). Eles também apuram e redigem de forma muito mais veloz do que os jornalistas e, por isso, podem ser escolhidos em coberturas onde a velocidade e a necessidade de atualização constante são essenciais. Na cobertura das eleições departamentais de 2015, por exemplo, o Le Monde usou uma plataforma de geração de textos automatizados para produzir 150 mil páginas na web em um período de 4 horas: ou seja, foram geradas 625 notícias por minuto (10 para cada segundo). Desta forma, foi possível fazer notícias sobre os 34 mil municípios e 2 mil cantões franceses (subdivisões administrativas), incluindo um com 35 habitantes. Uma tarefa, portanto, impossível de ser realizada pelos 70 jornalistas convocados pelo jornal francês, sendo que só 20 deles foram enviados por todo o país. Outro exemplo vem da própria Associated Press. Antes da automação, os jornalistas da AP faziam textos com 130 palavras, em média, que, em geral, eram disponibilizados na web de 15 a 20 minutos após a divulgação dos dados das companhias norte-americanas. Depois da automação, os textos aumentaram para 500 palavras, em média, e são postados apenas 1 minuto após a divulgação (LECOMPTE, 2015). O uso do software liberou 20 por cento do tempo da equipe da AP. Ele fez o trabalho que seria executado por três jornalistas com dedicação ao serviço em tempo integral.

Com mais tempo disponível, os jornalistas poderiam se dedicar às reportagens que demandam mais análise, contextualização, narrativas mais sofisticadas e criativas (DALEN, 2012; CARLSON, 2015; LECOMPTE, 2015). Os jornalistas também ficariam livres da tarefa de produzir notícias mais mecanizadas e repetitivas, como a checagem de dados em relatórios. Com base nessas possibilidades, Graefe (2016) aponta que ao profissional do futuro ficarão reservadas as tarefas que envolvem habilidades que os algoritmos não possuem, como análise em profundidade, entrevistas e reportagens investigativas. Lecompte (2015, p.35), uma entusiasta da automação de notícias, afirma que a IA vai liberar o jornalista para fazer o que realmente deve: contar histórias que realmente importam. 
Durante a revisão bibliográfica não foram identificadas notícias sobre demissões de jornalistas relacionadas à implantação da automação de notícias. Mas Latar (2015, p.67) sinaliza que os softwares serão uma forte competição para os jornalistas e Graefe (2016) aponta que alguns deles podem ser substituídos pelas plataformas de geração de texto na execução de tarefas mais repetitivas, que são comuns a muitos iniciantes na carreira. Em uma pesquisa feita na Coreia do Sul, executivos das empresas jornalísticas indicam que há uma tendência dos jornalistas serem demitidos nas redações que introduzirem a automação (KIM, D.; KIM, S., 2016, p.10). Outra possibilidade é a automação levar à redução de salários. Quanto melhor as máquinas forem capazes de substituir trabalhadores humanos, mais provável será que elas reduzam os salários dos trabalhadores com habilidades semelhantes. A lição de economia e estratégia de negócios é que você não quer competir com substitutos próximos, principalmente se tiverem vantagens competitivas. (BRYNJOLFSSON; MCAFEE, 2015, p. 203).

Por outro lado, algumas funções novas também podem ser criadas. Uma delas é a de editor de automação, que já está prevista no fluxo de trabalho da AP. Outra seria ocupada por jornalistas especializados no treinamento dos Agentes autônomos artificiais. Eles seriam responsáveis por estabelecer as regras das operações algorítmicas de produção de notícias: o que procurar nos dados, o que descartar, o que valorizar, que palavras devem ser usadas ou não, etc. Enfim, eles ensinariam os Agentes a seguirem os passos de um jornalista, mostrando quais são as regras básicas e os padrões essenciais do Jornalismo.

Os desafios do jornalismo com a produção de notícias automatizadas são grandes e é importante discutir usos, atribuições e consequências, pois como lembram Erik Brynjolfsson e Andrew McAfee $(2015,12)$, os problemas “não são intransponíveis" quando existe diálogo, mas as tecnologias "não vão reparar a si mesmas" sem a devida intervenção humana.

\section{CONSIDERAÇÕES}

Os últimos tempos revelam a crescente tecnologização do jornalismo, num ritmo cada vez mais veloz. Pode-se afirmar que a prática automatizada de apuração, redação e distribuição da notícia é a forma mais radical de uso da tecnologia até o momento, pois dispensa a presença humana do jornalista nestas etapas. A automatização de matérias jornalísticas, principalmente nas áreas financeira e esportiva, já é realidade em algumas redações da América do Norte, Europa e Ásia. Ela só foi possibilitada após a explosão de 
dados com a web; a partir de amplas formas de conexão e de compartilhamento; das ferramentas do Big Data, além do desenvolvimento de tecnologias ligadas à área da Inteligência Artificial.

Torna-se necessário ressaltar que apesar do avanço dos softwares de automação de texto, a questão sobre o que usar de fato e como usar, demanda debates amplos, pois seu uso tem implicações sociais. Constata-se que os Agentes autônomos artificiais produzem notícias mais rapidamente do que qualquer ser humano, o que contribui para culturas empresariais ligadas às ideias de eficiência e de corte de custo. Além disso, eles dispensam o jornalista de tarefas mais repetitivas para que eles possam se concentrar em textos sofisticados, analíticos e mais contextualizados. A notícia automatizada leva a mudanças nas rotinas produtivas das redações, cria novas funções, mas levanta preocupações sobre possíveis demissões e/ou redução do nível salarial dos jornalistas.

Apesar de não ser objeto do presente artigo, a automação de notícias requer debates éticos em relação aos julgamentos embutidos nos algoritmos e que não são conhecidos pelos consumidores da informação jornalística. Esse desconhecimento é ainda mais preocupante porque eles tendem a ver, conforme sinalizado por algumas pesquisas, as matérias produzidas pelos Agentes como conteúdo com mais credibilidade do que as realizadas pelos humanos. Outro ponto fundamental da automatização é que a oferta de conteúdos cada vez mais segmentados pode reduzir ainda mais o nível informacional dos consumidores de notícias sobre questões importantes que seus comportamentos na web indicam que não são de seus interesses. Por essas e outras questões, as implicações e consequências das notícias automatizadas precisam ser amplamente debatidas.

\section{REFERÊNCIAS}

ALJAZAIRI, Sena. Robot journalism: threat or an opportunity. Örebro University. 2016. Disponível em: <http://www.diva-portal.org/smash/get/diva2:938024/FULLTEXT01.pdf>. Acesso: 15 jun. 2016.

ANDERSON, C.W. Towards a sociology of computational and algorithmic journalism. New Media \& Society. 2013.p.1005-1021.

ARCE, Tacyana. O lead automatizado: uma possibilidade de tratamento da informação para o jornalismo impresso diário. Revista Exacta, Belo Horizonte, v.2, n. 3, 2009.

BARBOSA, Suzana. Jornalismo digital em base de dados (JDBD) - um paradigma para produtos jornalísticos digitais dinâmicos. 2007. Tese (Doutorado em Comunicação e Cultura Contemporâneas). Universidade Federal da Bahia, Salvador. Disponível em: $<$ http://migre.me/aTuYN $>$. Acesso em 10 abr. 2016. 
BRYNJOLFSSON, Erik; McAFEE. A segunda era das máquinas: trabalho, progresso e prosperidade em uma época de tecnologias brilhantes. Rio de Janeiro: Alta Books, 2015.

CARLSON, Matt. The robotic reporter: automated journalism and the redefinition of labor, compositional forms and journalistic authority. In: LEWIS, Seth C. (Org.). Digital journalism. v.3, n.3. New York: Taylor \& Francis Online, 2015.

CARREIRA, Krishma Anaísa Coura. Notícias automatizadas: a evolução que levou o jornalismo a ser feito por não humanos. 2017. Dissertação (Mestrado em Comunicação Social). Umesp.

CLERWALL, Christer. Enter the robot journalist: user's perception of automated content. In: Journalism practice. Special issue: future of journalism in an age of digital media and economic uncertainly. V.8, issue 5. New York: Taylor\&Francis Online, 2014.

COMTE-SPONVILLE, André. Dicionário filosófico. Tradução de Eduardo Brandão. São Paulo: Martins Fontes, 2003.

COSTA, Andriolli de Brittes da; SANTOS, Francisco dos. Reportagem algorítmica: imagens de um jornalismo sem jornalistas. In: II CONGRESSO INTERNACIONAL DO CRI2I, 29 a 31 out. de 2015. Porto Alegre.

COSTELLA, Antonio F. Comunicação: do grito ao satélite. Campos do Jordão: Mantiqueira, 2001.

DALEN, Arjen van. The Algorithms behind the headlines: How machine-written news redefines the core skills of human journalists. Journalism Practice. Volume 6, Issue 5-6. New York: Routledge, 2012.

DERTOUZOS, Michael. A revolução inacabada São Paulo: Futura: 2002.

DIAKOPOULOS, Nicholas. Algorithmic accountability reporting: on the investigation of black boxes. Town Center for Digital Journalism. 2013. Disponível em: < http://towcenter.org/wpcontent/uploads/2014/02/78524_Tow-Center-Report-WEB-1.pdf>. Acesso: 10 abr. 2016.

DÖRR, Konstantin Nicholas. Mapping the field of algorithmic journalism. 2015. Disponível em: <http://www.tandfonline.com/doi/full/10.1080/21670811.2015.1096748>. Acesso: 24 set.2016.

DUSEK, Val. Filosofia da tecnologia. Tradução de Luis Carlos Borges. São PauloLoyola, 2009.

EVANS, Dave. The internet of things: how the next evolution of the internet is changing everything. Cisco Internet Business Solutions Group. Apr. 2011. Disponível em: $<$ http://www.cisco.com/c/dam/en_us/about/ac79/docs/innov/IoT_IBSG_0411FINAL.pdf $>$. Acesso: 05 nov. 2015.

GLEICK, James. A informação: uma história, uma teoria, uma enxurrada. Tradução de Augusto Calil. São Paulo: Companhia das Letras, 2013.

GRAEFE, Andreas. Guide to automated journalism. Town Center for Digital Journalism. Jan. 2016. Disponível em: <http://towcenter.org/research/guide-to-automated-journalism/> Acesso em 08 abr. 2016.

et al. Perception of automated Computer-Generated news: credibility, expertise, and readability. Feb. 2016. Disponível em: < 
https://www.researchgate.net/publication/289529002_Perception_of_Automated_ComputerGenerated_News_Credibility_Expertise_and_Readability >. Acesso: 12 ago. 2016.

GUDWIN, Ricardo Ribeiro. (mensagem pessoal). Mensagem recebida por <krishmacarreira@gmail.com>em 6 de abr. 2016.

HAAK, Bregtje Van der; PARKS, Michael; CASTELLS, Manuel. The future of journalism: networked journalism. Internacional Journal of Communication. V.6, 2012.

JENKINS, Henry. Cultura da Convergência. Tradução de Susana L. de Alexandria. 2.ed. São Paulo: Aleph, 2009.

KAA, Hille van der; KRAHMER, Emiel. Journalist versus news consumer: the perceived credibility of machine written news. 2014. Disponível em: $<$ http://compute-cuj.org/cj2014/cj2014_session4_paper2.pdf > Acesso: 13 set. 2016.

KAKU, Michio. A física do futuro: como a ciência moldará o mundo nos próximos cem anos. Tradução de Maria Carvalho e João C. S. Duarte. Lisboa: Editorial Bizâncio, 2011.

KATO, Rafael. Conectar para crescer. Revista CEO Exame. São Paulo, n.22, p.52-59, nov. 2015.

KIM, Daewon; KIM, Seongcheol. Newspaper companies determinants in adopting robot journalism. Technological forecasting \& Social Change, 22 dez. 2016.

KURZWEIL. A era das máquinas espirituais. Tradução de Fábio Fernandes. 2.reimp. São Paulo: Aleph, 2007.

LATAR, Noam. Robot journalists: 'Quakebot' is just the beginning. Wharton Pennsylvania University. 2014.

. The robot journalism in the age of social physics: the end of human journalism? The New World of Transitioned Media. Springer, 2015.

LECOMPTE, Celeste. Automation in the Newsroom. Nieman Foundation, $1^{\circ}$. set. 2015. Disponível em: 〈http://niemanreports.org/articles/automation-in-the-newsroom>. Acesso em: 02 mar. 2016.

LINDEN, Car-Gustav. Decades of automation in the newsroom: why are there still so many jobs in journalism? Mar. 2016.

LOKOT; Tetyana; DIAKOPOULOS, Nicholas. News bots: automating news and information dissemination on Twitter. Digital Journalism, 2015.

MARCONI, Francesco; SIEGMAN, Alex. The future of augmented journalism: a guide for newsrooms in the age of smart machines. 2017. Disponível em:

<https://insights.ap.org/uploads/images/the-future-of-augmented-journalism_ap-report.pdf >. Acesso em: 10 ago. 2017

MARTINO, Luís Mauro Sá Martino. Teoria das mídias digitais. Linguagens, ambientes e redes. 2.ed. Petrópolis: Vozes, 2015.

MANOVICH, Lev. The language of new media. Massachusetts: MIT Press, 2001. 
NORMANDE, Naara. A automatização da narrativa jornalística. Estudos em Comunicação, n.13, p. 363-378, jun. 2013. Disponível em: < http://www.ec.ubi.pt/ec/13/pdf/EC13-2013Junho-14.pdf>. Acesso: 20 fev. 2016.

PRIMO, Alex. Transformações no jornalismo em rede: sobre pessoas comuns, jornalistas e organizações; blogs, Twitter, Facebook e Flipboard. Intexto: Revista do Programa de Pós-Graduação em Comunicação e Informação da Faculdade de Biblioteconomia e Comunicação da Universidade Federal do Rio Grande do Sul (UFRGS), Porto Alegre, n.25, p. 130-146, 2011.

RÜDIGER, Francisco. Tendências do jornalismo. 3.ed. Porto Alegre: UFRGS, 2003.

SANTOS, Márcio Carneiro dos. Narrativas automatizadas e a geração de textos jornalísticos: a estrutura de organização do lead traduzida em código. Brazilian Journalism Research: Revista da Associação Brasileira de Pesquisadores em Jornalismo (SBPJor), Brasília, v.12, n.1, 2016.

SAYURI, Juliana. Rumos do jornalismo. Estudo mostra a urgência de um modelo de negócio midiático adaptado à realidade digital. Revista Humanidades, revista do Centro de Ciências da Comunicação e Gestão da Universidade de Fortaleza. Jun. 2014. P. 82-85.

SQUIRRA, Sebastião. Jornalismo com convergências midiáticas nativas e tecnologias incessantes. In: FERREIRA JUNIOR, José; SANTOS, Márcio Carneiro dos (Orgs.). Comunicação, tecnologia e inovação. Porto Alegre: Buqui, 2013.

STRAUBHAAR, Joseph; LAROSE, Robert. Comunicação, mídia e tecnologia. Tradução de José Antonio Lacerda Duarte. São Paulo: Pioneira Thomson Learning, 2004.

WHITBY, Blay. Inteligência artificial: um guia para iniciantes. Tradução de Claudio Blanc. São Paulo: Madras, 2004.

WOLF, Mauro. Teorias da Comunicação. 1ed. Lisboa: Editorial Presença, 1987.

Original recebido em: 03 de junho de 2016

Aceito para publicação em: 28 de setembro de 2017

Sebastião Carlos de Morais Squirra

Doutor e mestre pelo Programa de Pós-Graduação em Ciências da Comunicação da Escola de Comunicação e Artes da Universidade de São Paulo. Fez Pós-doutorado nos EUA e na Espanha. Líder do Grupo de Pesquisa ComTec (Comunicação e Tecnologias Digitais). Atualmente, é Presidente da ABCiber (Associação Brasileira de Cibercultura).

Krishma Anaísa Coura Carreira

Doutoranda no Programa de Pós-Graduação em Comunicação Social da Universidade

Metodista de São Paulo, onde também é mestre com o tema "Notícias automatizadas: a evolução que levou o jornalismo a ser feito por não humanos". Pesquisa o cruzamento entre inteligência artificial e comunicação. Integra o grupo de pesquisa ComTec (Comunicação e Tecnologias Digitais).

Esta obra está licenciada sob uma Licença Creative Commons. 